\title{
Paternity testing and behavioral ecology: A case study of jaguars (Panthera onca) in Emas National Park, Central Brazil
}

\author{
Thannya Nascimento Soares ${ }^{1}$, Mariana P.C. Telles ${ }^{1,3}$, Lucileide V. Resende ${ }^{1}$, Leandro Silveira ${ }^{2,3}$, \\ Anah Tereza A. Jácomo ${ }^{2,3}$, Ronaldo G. Morato ${ }^{2,4}$, José Alexandre F. Diniz-Filho ${ }^{3,5}$, Eduardo Eizirik ${ }^{2,6}$, \\ Rosana P.V. Brondani ${ }^{7}$ and Claudio Brondani ${ }^{7}$ \\ ${ }^{1}$ Laboratório de Genética e Melhoramento, Instituto do Trópico Subúmido, \\ Universidade Católica de Goiás, Goiânia, GO, Brazil. \\ ${ }^{2}$ Associação Pró-Carnívoros, Atibaia, SP, Brazil. \\ ${ }^{3}$ Jaguar Conservation Fund, Mineiros, GO, Brazil. \\ ${ }^{4}$ CENAP-IBAMA, Atibaia, SP, Brazil. \\ ${ }^{5}$ Departamento de Biologia Geral, Instituto de Ciências Biológicas, \\ Universidade Federal de Goiás, Goiânia, GO, Brazil. \\ ${ }^{6}$ Laboratory of Genomic Diversity, NCI-Frederick, NIH, Frederick MD, USA. \\ ${ }^{7}$ Laboratório de Biotecnologia, Embrapa-CNPAF, Goiânia, GO, Brazil.
}

\begin{abstract}
We used microsatellite loci to test the paternity of two male jaguars involved in an infanticide event recorded during a long-term monitoring program of this species. Seven microsatellite primers originally developed for domestic cats and previously selected for Panthera onca were used. In order to deal with uncertainty in the mother's genotypes for some of the loci, 10000 values of $W$ were derived by simulation procedures. The male that killed the two cubs was assigned as the true sire. Although the reasons for this behavior remain obscure, it shows, in principle, a low recognition of paternity and kinship in the species. Since the two cubs were not very young, one possibility is that the adult male did not recognize the cubs and killed them for simple territorial reasons. Thus, ecological stress in this local population becomes a very plausible explanation for this infanticide, without further sociobiological implications.
\end{abstract}

Key words: ecological stress, infanticide, Jaguar, microsatellites, paternity.

Received: May 24, 2005; Accepted: April 10, 2006.

\section{Introduction}

Recent developments in molecular analyses have allowed the integration of genetics and ecology to elucidate complex ecological and behavioral phenomena at the population level, in the new field now called 'molecular ecology' (Beebee and Rowe, 2004). Molecular data have been used to discern overall patterns in population structure and to estimate demographic parameters, such as dispersion rates and inbreeding levels, making also possible a detailed analysis of some behavioral issues, such as mating systems and parentage (Frankham et al., 2003).

In Emas National Park (ENP, Goiás State, Central Brazil) in November 2001 two jaguar (Panthera onca)

Send correspondence to Mariana Pires de Campos Telles. Laboratório de Genética e Melhoramento, Instituto do Trópico Subúmido, Universidade Católica de Goiás. Caixa Postal 86, Av. Universitária 1440, 74605-010 Goiânia, GO, Brazil. E-mail: tellesmpc@uol.com. br. cubs, presumably nine months old, were killed and partially eaten by a $90 \mathrm{~kg}$ adult male jaguar, estimated to be eight years old. The two cubs were also radio-tracked since they were three months old and were definitely brother and sister. The identity of the killer was also conclusively identified through its radio-telemetry monitoring. This large male was being radio-tracked and his home range overlapped by $85 \%$ for a long time with the home-range of the cubs' mother and showed similar movements in time and space. During several days before the infanticide, the killer tracked movements of the female and the two cubs, which had been shifting their home ranges. Because of the large overlap of home ranges in space and time, this male was presumably the sire of the two cubs. This infanticide event was monitored by two of us (LS and ATAJ), during a long-term monitoring program of Jaguars in ENP.

However, in a subsequent capture event within the female's (mother) home range area, a previously unrecorded 
$105 \mathrm{~kg}$ adult male was caught and radio-collared. According to preliminary capture-recapture data, the population of Panthera onca in ENP is estimated as comprising about 15 specimens (Silveira, 2004). Finding another male overlapping the female's home range suggests that the observed infanticide could involve more complex sociobiological issues and introduce some uncertain in the paternity of the two killed cubs.

Since another male was found in the female's home range, the basic question becomes: who was the sire of the two cubs killed by the first captured male? We used microsatellite loci to test the paternity of the two $P$. onca males involved in the scenario described above and in this paper we discuss our results and the alternative sociobiological and ecological questions involved in the infanticide. To our knowledge, this is the first application of molecular data to address a behavioral ecology question in jaguars.

\section{Material and Methods}

Blood samples were obtained from the four jaguars involved in the case described above (the two cubs (C01 and $\mathrm{C} 02$ ) and the two adult males (M01, the killer and M02, the second male found in the area). No blood sample was available from the cub's mother. The blood samples were obtained using a Vacutainer ${ }^{\circledR}$ containing EDTA and Easy blood (TES) buffer (100 mM Tris, $100 \mathrm{mM}$ EDTA and 2\% SDS) and stored at $4{ }^{\circ} \mathrm{C}$. DNA extraction was performed using the GFX purification kit (Amersham Pharmacia Biotech $^{\mathrm{TM}}$ ) specific for blood. The extracted DNA was quantified and diluted to a concentration of $3 \mathrm{ng} / \mu \mathrm{L}$, to be used in the PCR reactions.

Ten microsatellite primers originally developed for domestic cats by Menotti-Raymond et al. (1999) and selected by Eizirik et al. (2001) for Panthera onca were used for the analyses: FCA005, FCA026, FCA077, FCA090, FCA096, FCA126, FCA193, FCA211, FCA220, FCA441. In this specific case, we were able to obtain good amplification products for seven of the ten primers, following the original protocol in Eizirik et al. (2001). Allelic polymorphism was detected in $4 \%(\mathrm{w} / \mathrm{v})$ denaturing polyacrylamide gel and visualized by silver staining (Bassam et al., 1991).

After scoring, the genotypes of the seven loci and the parentage index $W$ was calculated according to Weir (1996) and Evett and Weir (1998). This $W$ index measures the inclusion probability, for each of the two males, and is given as

$$
W=\frac{p r L}{(p r L+1-p r)}
$$

where $p r$ is the a priori probability of an alleged sire being the true sire, and $L$ is the likelihood of the paternity, i.e., the probability of the genotype of a child "O" given that he/she descends from a mother $\mathrm{M}$ and the tested sire, divided by the probability of "O" descending from mother $\mathrm{M}$ and any other sire $\mathrm{P}$ in the population, in such a way that

$$
L=\frac{\operatorname{Pr}(O \mid M, P t)}{\operatorname{Pr}(O \mid M, P)}
$$

For example, the value of $L$ for a given cub with genotype Aa for a given locus, son of a mother AA with an alleged sire with a genotype Aa is given by

$$
L=\frac{\frac{1}{2}\left(P^{2}\right)(2 p q)}{q\left(p^{2}\right)(2 p q)}=\frac{p^{3} q}{2 p^{3} q^{2}}=\frac{1}{2 q}
$$

where $p$ and $q$ are the frequencies of the alleles $\mathrm{A}$ and a in the population (Weir, 1996). When many loci are analyzed simultaneously, an overall $L$ value is obtained as the product of the $L$ value from all loci. So, if a single locus excludes the possibility of paternity, the overall likelihood goes to zero. Assuming that there is no 'a priori' reason for supporting paternity of an alleged sire, $\mathrm{pr}=0.5$, in such a way that $W$ becomes simply

$$
W=\frac{L}{L+1}
$$

In this study, this simply theoretical reasoning becomes more complicated due to some uncertainty in several parameters. Firstly, the genotype of the mother could not be derived with certainty from the genotypes of the two cubs for four of the seven loci used (see the 'results' section). Secondly, there were no precise estimates of the allele frequencies of this local population, although overall frequencies estimated from data for 44 jaguars across the geographic range of the species are available (see Eizirik et al., 2001) and were used here as an initial approximation.

To deal with all these uncertainties, 10000 values of $W$ were derived by simulation procedures, after randomly adding up to $20 \%$ of uniform variation around the average allele frequencies estimated using the original data of Eizirik et al. (2001) and combining all possible genotypes of the mother for each locus given the genotypes of the cubs. The probability of occurrence of each genotype was also estimated by genotypic frequencies from the data of Eizirik et al. (2001). The 10000 values of $W$ were also estimated using only the three loci definitely known as belonging to the mother's genotype, which included only the uncertainty in the allele frequency estimates.

These procedures were performed for each cub, and the joint probability of paternity in the simulations was calculated from the product of the probabilities for each cub. Simulations were performed in software written in QBASIC and designed specifically for this study.

\section{Results}

The seven primers with a good amplification pattern provided a system with seven loci and up to three alleles per 
locus to test the paternity (Figure 1). After coding the genotypes (Figure 1) it was possible to see that the M02 male was automatically excluded by three out of the seven loci (FCA077, FCA193 e FCA441), independently of the mother's genotype, since its likelihood tended to zero (except if one assumes that mutation and coding errors were frequent for all three loci simultaneously).

To establish the probability that the M01 male is the true sire it was necessary to use the mother's genotypes (Table 1), which could be determined exactly for only three loci, after combining the genotype of the two cubs which were almost certainly sister and brother according to field observations. Taking into account only the uncertainty in allele frequencies ('A) the distribution of the $10000 \mathrm{~W}$ values peaked at 0.9943 and ranged from 0.990 to 0.997 for each individual cub. This average probability value reduced to 0.988 and ranged from 0.982 to 0.993 when both cubs were considered simultaneously (Figure 2B). Overall, the probability was very high that the M01 male was the true sire of the two cubs.

To use the seven loci, the simulation procedures were repeated, but randomly using all possible mother's genotypes, based on their relative frequency in the population estimated using the original data of Eizirik et al. (2001). However, for the FCA096 and FCA220 loci at least one of the possible genotypes for the mother also excluded the M01 male as the true sire. So, in this case, neither of the males could have sired the cubs. However, Bayesian inference can be used to solve this apparent paradox if the frequencies of these genotypes is known and the probability of paternity given by the loci from which the mother's genotype if known without doubt.

In relation to other possible genotypes for the mother, the estimated frequency of excluding genotypes of the mother was initially established as 0.25 , for the first cub, in such a way that $L$ values would go to zero in this proportion of simulations. However, it can be assumed that this possibility could invalidate all previous results using only loci for which the genotype of the mother is known with certainty. According to Bayesian reasoning, suppose that $H_{0}$ is the hypothesis that the M01

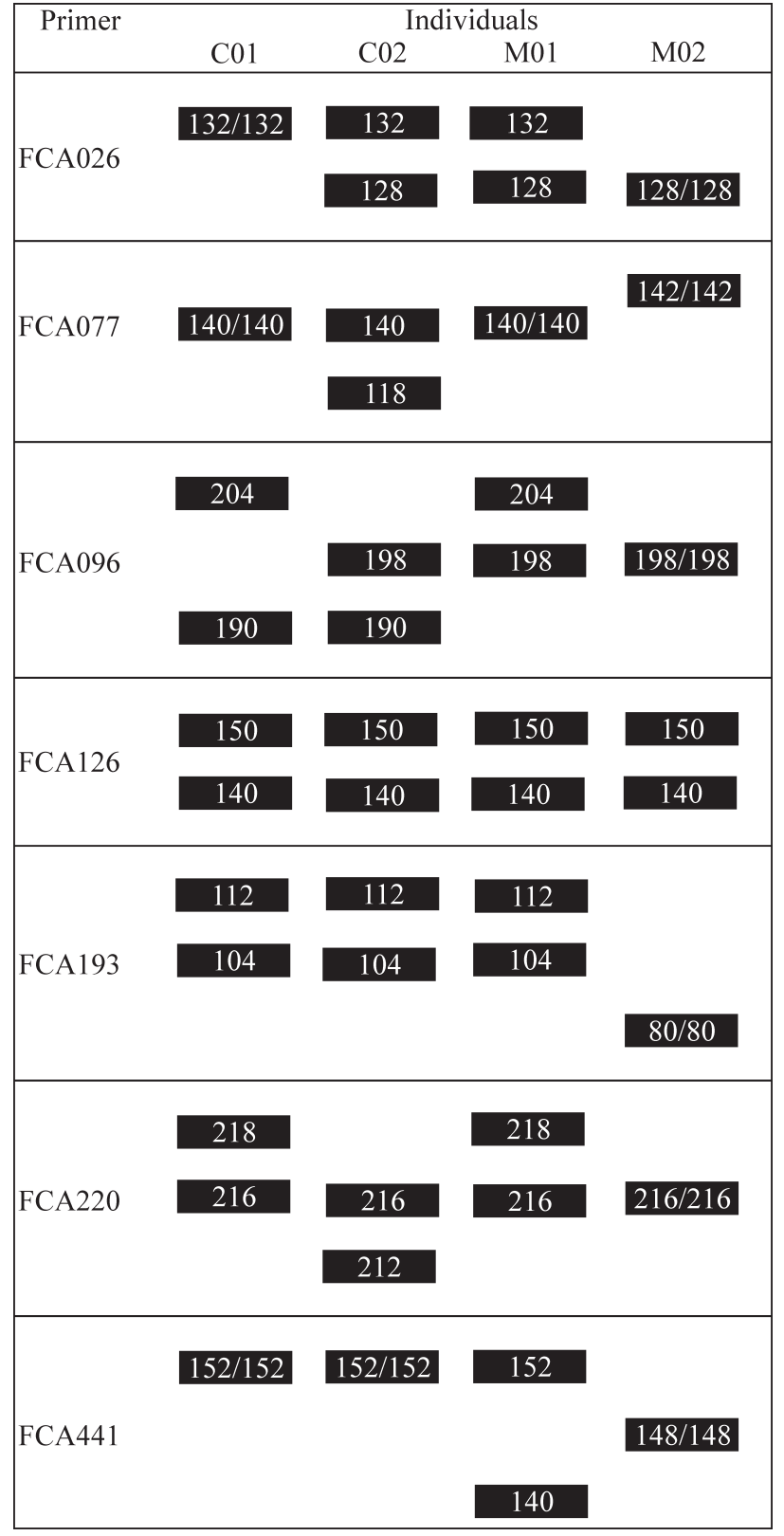

Figure 1 - Genotypes of four jaguars (Panthera onca) obtained using the FCA026, FCA077, FCA096, FCA126, FCA193, FCA220 e FCA441 primers.

Table 1 - Genotypes observed at seven microsatellite loci for the two cubs (C01 and C02 and two possible sires (M01 and M02) and the possible mother's genotypes derived from the genotypes of the two cubs.

\begin{tabular}{lccccc}
\hline Loci & C01 & C02 & M01 & M02 & Mother (possible genotypes) \\
\hline FCA026 & $132 / 132$ & $132 / 128$ & $132 / 128$ & $128 / 128$ & $132 / 128$ \\
FCA077 & $140 / 140$ & $140 / 118$ & $140 / 140$ & $142 / 142$ & $140 / 118$ \\
FCA096 & $204 / 190$ & $198 / 190$ & $204 / 198$ & $198 / 198$ & $204 / 198^{*} 204 / 190190 / 198190 / 190$ \\
FCA126 & $150 / 140$ & $150 / 140$ & $150 / 140$ & $150 / 140$ & $150 / 150150 / 140140 / 140$ \\
FCA193 & $112 / 104$ & $112 / 104$ & $112 / 104$ & $80 / 80$ & $112 / 114112 / 112114 / 114$ \\
FCA220 & $218 / 216$ & $216 / 212$ & $218 / 216$ & $216 / 216$ & $218 / 216^{*} 218 / 212216 / 216^{*} 216 / 212$ \\
FCA441 & $152 / 152$ & $152 / 152$ & $152 / 140$ & $148 / 148$ & $152 / 152$ \\
\hline
\end{tabular}

*The mother's genotypes which, if observed, would exclude both sires. 

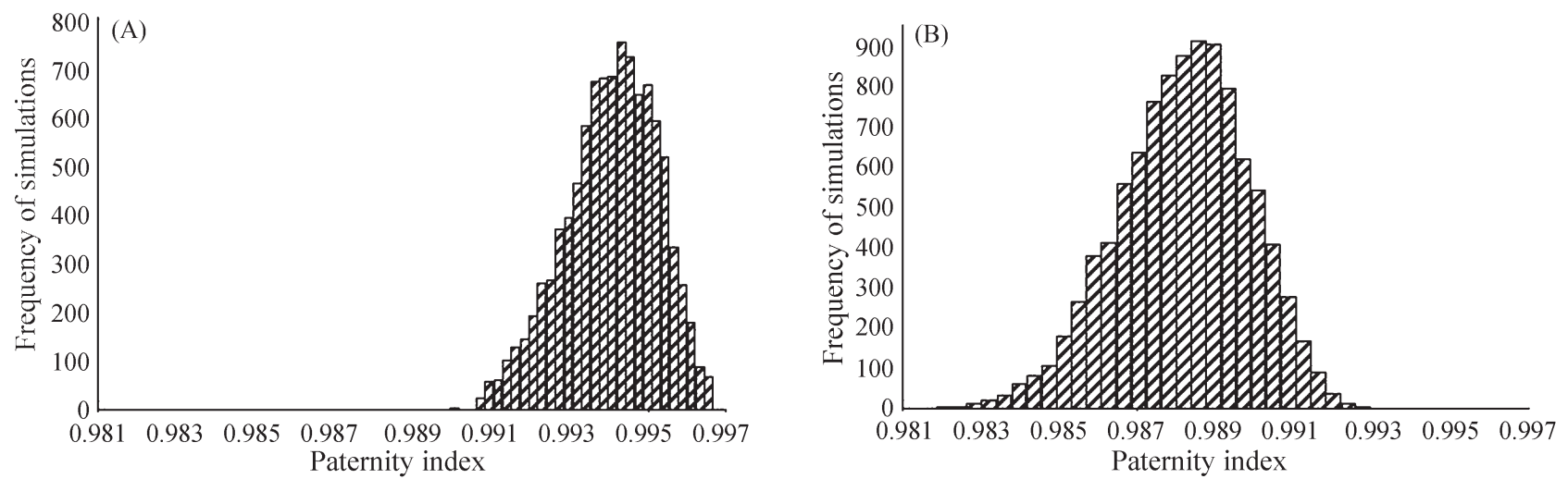

Figure 2 - Frequency distribution of 10000 values for the paternity index of the M01 male for each cub (A) and both cubs simultaneously (B), considering only the three loci for which the genotype of the mother was known and taking into account the uncertainty in the allele frequency estimates.

male is the true sire of the two cubs, as strongly supported by the three loci, and that $H_{l}$ is the alternative hypothesis. The average estimated probability of $H_{0}$ using only the three loci was 0.994 and, consequently, the probability of $H_{1}$ was 0.006 . Considering the four possible primer FCA096 genotypes for the mother there would be a $25 \%$ a priori chance of rejecting $H_{0}$. However, a posteriori $(\mathrm{Pp})$ probability of maternal genotype that exclude the sire becomes, according to Bayesian formulation (Hilborn and Mangel, 1997), $\mathrm{Pp}=(0.25 \mathrm{x}$ $0.006) /[(0.25 \times 0.006)+(0.75 \times 0.994)]=0.002(0.2 \%)$. This genotype would occur in only $0.20 \%$ of the simulations so that the overall likelihood equals zero. For the second cub, this a posteriori probability, calculated using the same reasoning, was slightly larger $(0.32 \%)$.

The simulation procedures, considering the seven loci and uncertainty in both allele frequencies and the genotype of the mother (Figure 3), furnished a left-skewed $W$ distribution with values for both cubs highly concentrated near one. Thus, even under all uncertainty, the median probability that the M01 male was the sire of the two cubs was higher than $99 \%$.

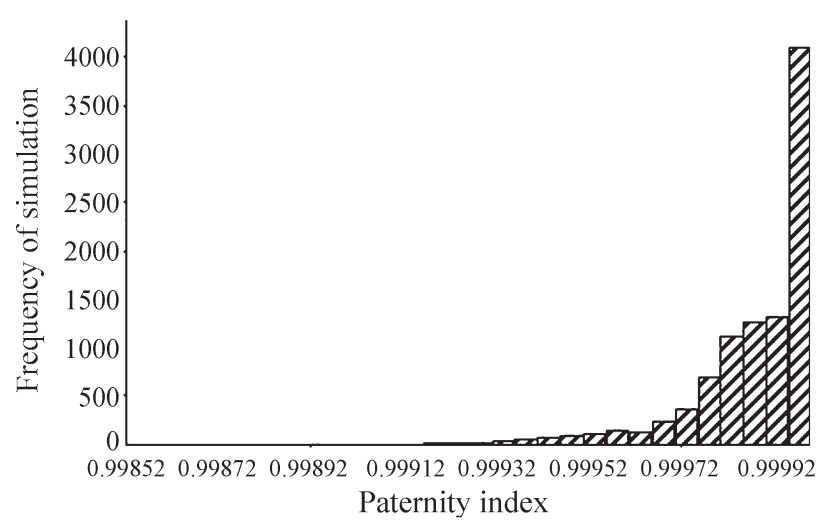

Figure 3 - Frequency distribution of 10000 values for the paternity index of male the M01 male for both cubs simultaneously, considering the seven loci and taking into account uncertainty both in the genotype of the mother and the allele frequency estimates.

\section{Discussion}

\section{The paternity test and the ecological scenario}

The paternity test performed here strongly indicated that the M01 male that was being radio-tracked and had followed and killed the two sister cubs was actually their sire. We cannot be absolutely certain that the M01 male, and not a close relative, was the true sire of the two cubs. However, our field observations and overall knowledge of jaguar ecology suggest a very low jaguar population density in this region (Silveira, 2004), so there would be a very limited number of males close to this female and her cubs. Indeed, finding the M02 male in the area was the very motive that triggered the paternity investigation presented here.

If the M02 male had been assigned as the true sire of the two killed cubs, the scenario would be, in principle, a classical sociobiological event in which a new male (in this case, the M01 male) entering the area would try to kill the two cubs in order to release their mother for mating. However, at approximately 75 days of age, jaguar cubs begin taking solid meat, with suckling lasting for only about five months. Thus, it is possible that females resume cycling 2-3 weeks after lactational anestrus, as reported for the domestic cat (Oliveira, 1994). In addition, jaguars are not seasonal breeders (Morato et al., 2004) and thus quickly return to cycling after lactational anestrus. In this way, it is possible that the mother had been free to mate for at least four months before the infanticide, which could help to exclude this first scenario. More importantly, since the genetic analyses showed that the M01 male (the killer) was the true sire with a very high probability, more complex sociobiological and ecological conditions for the event must be considered.

It is important to note that telemetry data revealed that the M01 male did not simply find the cubs, kill and eat them but that it followed the mother and the cubs for a few days before the infanticide. The reasons for this behavior remain obscure but may reflect a low recognition of paternity and kinship in the species, although previous papers have proposed that shifts in the home ranges of females (and associ- 
ated cubs) may be a strategy to avoid infanticide in mammalian Carnivora (see Dahle and Swenson, 2003). Since the two cubs were not very young when they were killed, this could represent a competition issue, in that the adult male did not recognize the cubs as his offspring and killed them for simple territorial reasons when under ecological stress.

Although ENP is a relatively large national park (132,000 hectares), only about $40 \%$ of this area includes very suitable jaguar habitat and therefore competition among individuals for food and mates may be higher than observed in a large, continuous and well-preserved population (Silveira, 2004). Maximum jaguar home ranges can be very large in some instances, up to 18,000 hectares (Kelt and Van Vuren, 2001; Diniz-Filho et al., 2005), so that only 2 or 3 males would be found in the area with minimum home range overlap. This is a little higher than our findings using preliminary capture-recapture data using camera traps (see Silveira et al., 2003), which suggested a total population of 10-12 jaguars (about half of these would be males) in the ENP region and surroundings (Silveira, 2004). Population density in the ENP would be thus a little higher than expected. Another factor is that the region surrounding the park is highly fragmented and occupied by large soybean plantations, which probably limit jaguar dispersion and migration. Thus, ecological stress becomes a plausible explanation for this infanticide, without further complex sociobiological implications (Sandell, 1989). It is also interesting to note that this scenario would lead to high levels of inbreeding, that still need to be investigated in this particular population. However, a first insight towards supporting relatively high levels of inbreeding in this particular population would start by noticing that one of the individuals genotyped, the excluded parent M02, is homozygous for six out of the seven loci analyzed here.

Infanticides, including those committed by females, are not rare in mammals (Hausfater and Hardy, 1984; Tuomi et al., 1997; Ebensperger, 1998; Dobson et al., 2000), with non-parental infanticide having usually been considered to be an adaptive behavioral strategy to enhance the reproductive success of the aggressor (Agrell et al., 1998). In some circumstances, infanticide can become an evolutionary stable strategy (ESS; Tuomi et al., 1997; Ebensperger, 1998). Although parental infanticide is usually expected to reduce individual fitness, it is important to consider that other complex ecological and demographic components may be associated with this behavior (Pusey and Packer, 1994; Wolff, 1997; Wolff and Peterson, 1998; Whitman et al., 2004).

Due to their carnivorous habits and because most species bear altricial young, carnivores are more likely to exhibit infanticide than any other mammalian order. However, the reasons that lead to infanticide are still fairly unclear for most species (see reviews by Hausfater and Hardy, 1984; Tuomi et al., 1997). Although infanticide has been recorded for almost all large cats from the Panthera group, distinct sociobiological interpretations are discussed for these species (see Davies and Boersma, 1984; Bailey, 1993; Smith, 1993). Besides an expected natural rate of infanticide among free-living populations, it is also expected that stressful environmental conditions may favor abnormal infanticide rates in solitary carnivores (Sandell, 1989) and infanticide is sometimes considered to be part of the mammalian population regulation process (Wolff, 1997). In the case of Emas National Park presented here, we are more prone to believe that the isolation of the local jaguar population may be leading to stress-based infanticide behavior among male jaguars that are disputing the few available females.

\section{Methodological issues}

With respect to methodological issues, the analyses performed using simulation procedures were intended to evaluate how the uncertainty in mother genotypes (for four out of the seven loci used) and allele frequencies could qualitatively affect decisions about paternity.

The uncertainty about the genotype of the mother could introduce a problem, since one of the possible genotypes, for the $\mathrm{C} 01 \mathrm{cub}$, would also exclude the paternity of the M01 male. Because frequencies of all four possible genotypes are very low in the data of Eizirik et al. (2001) we assumed that the probability of this excluding genotype would be 0.25 . However, under Bayesian reasoning this high probability is only the 'a priori' probability and it is also necessary to take into account previous results using the three loci for which genotype of the mother is known for sure, which means that the a posteriori probability of having these genotypes is much less than 0.01 .

The other main source of error in our analyses is the lack of knowledge of allele frequencies in the studied population. We used as initial values of allele frequencies for stochastic simulations the overall data for 44 individuals distributed throughout the range of the species. It would also be possible to restrict allele frequency estimates to the 17 jaguars from the South America and southern region in relation to the Amazon River (the southern clade) described by Eizirik et al. (2001), but this would reduce the sample size a lot. Anyway, the $F_{S T}$ values estimated for microsatellites by Eizirik et al. (2001) were always smaller than 0.085 (although a significant phylogeographic structure was detected using mtDNA sequences). Also, although the $20 \%$ error assumed for allele frequencies in the simulations was arbitrary, our results with higher error values of up to $50 \%$ (not shown) did not qualitatively change the main conclusions, with median paternity indexes always higher than $99 \%$.

Thus, by considering these three main methodological points, there is a high probability that the M01 male was the sire of the two killed cubs, and this conclusion is not qualitatively affected by uncertainties in the data. The 
sociobiological and ecological conditions that triggered this infanticide remain unclear, but our analyses show that under the conditions prevalent in the Emas National Park even the probable sire of a cub can become that cubs killer, with obvious consequences for the maintenance and conservation of the jaguar populations in the area. In a more general sense, our analyses demonstrate the use of statistical approaches to assess and improve the power of molecular methods in behavioral studies in cases when uncertainties are present in the data set.

\section{Acknowledgments}

We thank K.M. Pellegrino and two anonymous reviewers for helpful comments that greatly improved previous versions of the manuscript. We are also thankful to the Brazilian Institute of the Environment and Renewable Natural Resources (Instituto Brasileiro do Meio Ambiente e dos Recursos Naturais Renováveis - IBAMA) authorities at Emas National Park for permission to carry out this jaguar research, to the Centro Nacional de Pesquisa para a Conservação de Predadores Naturais (CENAP/IBAMA) and the Associação Pró-Carnívoros for financial support. We further thank M. Menotti-Raymond, V. David and S.J. O'Brien (LGD, NCI, USA) for access to the microsatellite primers and to $\mathrm{CNPq}$ for doctoral and researcher scholarships to LS, ATAJ and JAFD-F.

\section{References}

Agrell J, Wolff JO and Ylonen H (1998) Counter-strategies to infanticide in mammals: Costs and consequences. Oikos 83:507-517.

Bailey TN (1993) The African Leopard: Ecology and Behavior of a Solitary Felid. Columbia University Press, New York, 429 pp.

Bassam BJ, Caetano-Anolles G and Gresshoff PM (1991) Fast and sensitive silver staining of DNA in polyacrilamide gels. Analytical Biochemistry 196:80-83.

Beebee T and Rowe G (2004) An Introduction to Molecular Ecology. Oxford University press, Oxford, 346 pp.

Dahle B and Swenson JE (2003) Seasonal range size in relation to reproductive strategies in brown bears Ursus arctus. J Anim Ecol 72:660-667.

Davies EM and Boersma PD (1984) Why lionesses copulate with more than one male. Am Nat 123:594-611.

Diniz-Filho JAF, Carvalho P, Bini LM and Tôrres NM (2005) Macroecology, geographic range size - Body size relationship and minimum viable population analysis for new world carnívora. Acta Oecol 27:25-30.

Dobson FS, Chesser RK and Zinner B (2000) The evolution of infanticide: Genetic benefits of extreme nepotism and spite. Ethol Ecol Evol 12:131-148.

Ebensperger LA (1998) Strategies and counterstrategies to infanticide in mammals. Biol Rev 73:321-346.
Eizirik E, Kim J, Menotti-Raymond Jr M, Crawshaw PG and O’Brien SJ (2001) Phylogeography, population history and conservation genetics of jaguars (Panthera onca, Mammalia, Felidae). Mol Ecol 10:65-79.

Evett IA and Weir BS (1998) Interpreting DNA Evidence: Statistical Genetics for Foresinc Scientists. Sinauer, Sunderland, $278 \mathrm{pp}$.

Frankhman R, Ballou JD and Briscou DA (2003) Introduction to Conservation Genetics. Cambridge University Press, Cambridge, $617 \mathrm{pp}$.

Hausfater G and Hardy SB (1984) Infanticide: Comparative and Evolutionary Perspectives. Aldine Publishing Company, New York, 598 pp.

Hilborn R and Mangel M (1997) The Ecological Detective: Confronting Models with Data. Princeton University Press, New Jersey, $315 \mathrm{pp}$.

Kelt DA and Van Vuren DH (2001) The ecology and macroecology of mammalian home range area. Am Nat 157:637645.

Menotti-Raymond M, David VA, Lyons LA, Schaffer AA, Tolmlin JF, Hutton MK and O'Brien SJ (1999) A genetic linkage map of microsatellites in the domestic cat (Felis catus). Genomics 57:9-23.

Morato RG, Verreschi ITN, Guimarães MABV, Cassaro K, Pessuti C and Barnabe RC (2004) Seasonal variation in the endocrine-testicular function of captive jaguar (Panthera onca). Theriogenology 68:1273-1281.

Oliveira TG (1994) Neotropical Cats: Ecology and Conservation. Edusma, São Luís, 220 pp.

Pusey AE and Packer C (1994) Non-offspring nursing in social carnivores - Minimizing the costs. Behav Ecol 5:362-374.

Sandell M (1989) The mating tactics and spacing patterns of solitary carnivores. In: Gittleman JL (ed) Carnivore Behavior, Ecology and Evolution. Cornell University Press, New York, pp 132-154.

Silveira, L (2004) Ecologia comparada de onça-pintada (Panthera onca) e onça-parda (Puma concolor) no cerrado do Brasil. Doctoral Thesis, Universidade de Brasília, Brasília.

Silveira L, Jácomo ATA and Diniz-Filho JAF (2003) Cameratrap, line transect census and track surveys: A comparative evaluation. Biol Conserv 114:351-355.

Smith JLD (1993) The role of dispersal in structuring the Chitwan tiger population. Behaviour 124:165-195.

Tuomi J, Agrell J and Mappes T (1997) On the evolutionary stability of female infanticide. Behav Ecol Sociobiol 40:227233.

Weir BS (1996) Genetic Data Analysis II: Methods for Discrete Population Genetic Data. Sinauer Associates, Sunderland, $445 \mathrm{pp}$.

Whitman K, Starfield AM, Quadling HS and Packer C (2004) Sustainable trophy hunting of African lions. Nature 428:175-178.

Wolff JO (1997) Population regulation in mammals: An evolutionary perspective. J Anim Ecol 66:1-13.

Wolff JO and Peterson JA (1998) An offspring-defense hypothesis for territoriality in female mammals. Ethol Ecol Evol 10:227-239.

Associate Editor: Horácio Schneider 